\title{
Emotional and Behavioural Disorders in Children and Adolescents with Neoplasm
}

\author{
Mohammad S.I. Mullick ${ }^{1}$, Sultana Algin ${ }^{2}$ \\ ${ }^{1}$ Professor of Child and Adolescent Psychiatry and Chairman, Department of Psychiatry, BSMMU, Dhaka, ${ }^{2}$ Assistant Professor, Department \\ of Psychiatry, BSMMU
}

\begin{abstract}
:
Background: Chronic physical illness including neoplasm has significant adverse impact on quality of life of the effected children and adolescents and their caregivers. This aspect has yet not been explored in Bangladesh and there exists significant lack of awareness. Objectives: To delineate the frequency of emotional and behavioural disorders among children and adolescents with neoplasm and to find out the relationship of socio-demographic and relevant variables with psychiatric disorders of them. Methods: This was a cross sectional study conducted at Department of Psychiatry, Bangabandhu Sheikh Mujib Medical University, Dhaka. Sixty children and adolescent with neoplasm admitted in paediatric unit of same institution who were referred to psychiatric department for assessment. They were assessed for emotional and behavioural disorders by using validated Bangla translation of a standardized child psychiatric assessment tool, the Development and Well-Being Assessment. Results: Out of 60 cases, 53\% had been suffering from any ICD-10 psychiatric disorders (87.5\% emotional and 34.7\% behavioural disorders). Seventy five percent of children with ALL, 18\% with Hodgkin Lymphoma followed by 7\% with Wilms' tumour suffered from psychiatric disorders. Emotional and behavioural disorder was found more in ALL cases. Though emotional disorder was found higher among girls, the difference was not significant. Conversely, behavioural disorders were found among the boys which was highly significant $(\mathrm{p}<0.005)$. In terms of education, both emotional and behavioural disorders were significantly found in higher grade. Conclusion: The identification and management of the emotional and behavioural disorders that accompany chronic physical illness including neoplasm would optimize treatment outcome and quality of life.
\end{abstract}

Key words: Emotional \& behavioural disorders, chilodren \& adolescents, neoplasm.

[BSMMU J 2010; 3(2): 86-90]

\section{Introduction}

Chronic physical illness including neoplasm has significant adverse impact on quality of life of children and adolescent. Leukemia is the most common pediatric malignancy; it accounts for $39 \%$ of all childhood cancers .It is also evident that cancer is the number one cause of death due to disease in children aged one through adolescence ${ }^{1}$. However, cancer is no longer equated with death, as it was in the past. As new medical treatments and technologies are developed, more and more children in each year are survivors of cancer; over $60 \%$ of children with cancer can survive. These children are able to continue normal lives during and after treatment ${ }^{2}$.

Chronic illnesses in children and adolescents have devastating influence on them and their families. The patients have to cope up with illness, medication and its influence on their development. Consequently, a large

Address for Correspondence: Prof. Mohammad S.I. Mullick. Professor of Child and Adolescent Psychiatry and Chairman, Department of Psychiatry, BSMMU, Email address: msimullick@ gmail.com number of them have emotional disorders that influence the course and outcome of physical disorder. In a prominent hospital based study, it was found that $90 \%$ children with acute lymphoblastic leukemia had emotional disorders. All the disorders were mild to moderate in intensity and were perceived to be easily treatable ${ }^{3}$.In other representative studies, findings revealed that a majority of attending parents of children with acute lymphoblastic leukemia suffered from depression, adjustment disorder ${ }^{4,5}$. In general, a poor body image is associated with self-rankings of academic, social, and psychological impairment, low self-esteem, and symptoms of depression. In a representative study on psychological consequences of chronic physical illness, it was found that children with cancer or a cancer survivor experienced different kinds of psychiatric problems in different aspect of their life. Researchers have found a variety of these psychological difficulties in children affected by cancer. These reactions and conditions included anxiety and panic (both chronic and acute), inhibited and withdrawn behavior, fear of trying new things, low emotional 
expressiveness, behavior problems, an excessive number of unexplained somatic complaints, intense stress, posttraumatic stress disorder, frustration and discouragement related to school difficulties, peer relationship difficulties, loss of independence (especially during adolescence), concern about ability to attract the opposite sex, and worries about the future in relation to career and relationships ${ }^{1}$. It has been stated that "the chronic strains of childhood cancer, such as treatment-related pain; nausea and vomiting; visible side effects such as hair loss, weight gain or loss, and physical disfigurement; and repeated absences from school and peers, interact to negatively impact social and psychological adjustment"1. Contributing to the learning problems which many students with cancer face is the high rate of absenteeism that may result from hospitalizations, treatments, and treatment side effects ${ }^{1}$.With the advancement of medical science, survival rate of cancer patients has been increased. Psychiatric co-morbidity of children and adolescents and their parents is also treatable. This study was aimed to delineate the frequency of emotional and behavioral disorders among children and adolescent with neoplasm and to find out the relationship of socio-demographic and relevant variables with psychiatric disorders among them. As this era has not been explored before in Bangladesh context, we should stand beside our future generation

\section{Methods}

This was a cross-sectional study conducted from July 2008 to June 2009 at the department of Psychiatry, Bangabandhu Sheikh Mujib Medical University (BSMMU). Sixty children and adolescents with neoplasm admitted in paediatric unit of BSMMU who were referred to psychiatric department for assessment. The instruments that were used in this study were as following:

a) A semi-structured questionnaire was used that included socio-demographic characteristics, family type and relevant variables including information about neoplasm, designed for the study. Prior use, the items of the questionnaire were pretested, finalized for collecting data.

b) Development and Well-Being Assessment (DAWBA): The assessment of the emotional and behavioral disorders was carried out using the DAWBA ${ }^{6}$. It is an internationally well accepted research instrument and novel package of questionnaire, interviews and rating techniques designed to generate ICD-10 and DSM-IV psychiatric diagnoses among children and adolescents of 5 to 16 years $^{6}$. It primarily focuses on the common emotional, behavioral and hyperactivity disorders and also covers less common disorders more briefly. It has three versions- parent version, self version and teacher version, its parent version was used for all cases. This uses a mixture of closed and open questions about child psychiatric symptoms and their impact (i.e. resultant distress and social impairment). The interview are administered by interviewers, who also record verbatim accounts of any reported problems, but do not rate them. Experienced clinicians subsequently review both the verbatim accounts and the answers to structured questions before assigning diagnoses. This instrument has been translated in Bangla, standardized and validated ${ }^{7}$. Validated Bangla DAWBA was used in this study for measuring child psychopathology of the cases.

c) ICD-10(DCR): It is the $10^{\text {th }}$ revision of International Classification of Diseases by the World Health Organization which is also used for diagnostic criteria for the research ${ }^{8}$. It provides the specific criteria for the diagnoses of mental and behavioral disorders. In this study only Axis-1 diagnoses of ICD-10 were considered and DAWBA generated diagnosis was assigned according to Axis 1 of ICD-10.

After taking informed consent, trained research assistant interviewed parents of cases by using questionnaire for socio-demographic characteristics and relevant variables. The parents of all cases were interviewed by using parent version of validated Bangla translation of a standardized child psychiatric assessment tool DAWBA. Then the diagnosis was assigned according to ICD-10DCR.Case who had at least one or more Axis-1 diagnosis of psychiatric disorder was cosidered as having 'any disorder'. Comorbid diagnoses in Axis-1 disorder were recorded for the analysis for specific psychiatric disorder and also for the analysis of disorders on the basis of broad diagnostic categories. The data was computed into the computer based diagnostic programme for generating computer based diagnosis. It was rated by the principal author of the study, who was an internationally recognized and experienced rater for the DAWBA diagnoses. Statistical analysis was carried out using the SPSS package. Chi-square test was used to measure the level of significance of any independent association between the qualitative variables. Significance test was done at 95\% confidence interval. A p value of $\leq 0.01$ was considered to be significant.

\section{Results}

Analysis was made on 60 cases in the present study. Sample characteristics revealed that 34(57\%) cases were boys and 26(43\%) cases were girls giving a boy: girl ratio 
of 1.3:1. Their age ranged from 5 to 16 years with a mean age of $9.93( \pm 3.4)$ years. The age group of 5-10 years was most prevalent with 32(53.3\%) cases. Thirty five (58.3\%) cases came from nuclear family, 31(51.7\%) cases were of middle income group and 38(63.3\%) cases were with urban background. Considering level of education, 38(63.3\%) cases were in class I-V, 13(21.7\%) cases were in class VIXI. Out of 60 cases, the most prevalent neoplasm was found ALL with 45(75\%) cases followed by 11(18\%) cases with Hodgkin lymphoma and the rest 4(7\%) with Wilm's tumor (Fig-1). Any forms of psychiatric disorders were found in 32(53.3\%) cases. Analysis of the group of disorders was made and co-morbidity was included in the analysis that caused increase of the number of cases with psychiatric morbidity. It revealed that 28(87.5\%) cases had emotional disorders and 11(34.7\%) cases had behavioral disorders (Table-1). Further, specific type of disorders was analyzed. Of the emotional disorders, specific phobia and other anxiety disorders were most prevalent (25\% for each) followed by generalized anxiety disorder and major depressive disorder (14\% for each). Of the behavioral disorders, hyperkinetic disorder (45\%), oppositional defiant disorder (36\%) was found (Table-2). Comparative analysis of the three groups of patients with neoplasm having psychiatric diagnoses revealed that the first place was taken by the children and adolescents with ALL (73\%). Fig.II shows that Emotional and behavioral disorder found more in children with ALL than that of other types of neoplasm. The defrences did not reach the level of signifiance ( $p>0.05)$. Fisher's exact test was done for the analyses of association between psychiatric diagnoses and socio-demographic characteristics (Table-III). It revealed that no significant difference of variables was found except age group and level of education. Psychiatric disorder was found significantly higher among 11-16 year age group and grade VI-XI educational level $(\mathrm{p}<0.05$ for both). Further analysis was done between sociodemographic characteristics and broad category of psychiatric disorder that has been presented in Table-IV. It was found that both emotional and behavioural disorder were not statistically significant between age groups. Though Emotional disorder was found higher among girls than that of boys but the difference did not reach the level of significance. Conversely, behavioral disorder was found higher among the boys than that of girls which was highly significant $(p<0.005)$. In terms of education, both emotional and behavioral disorders were found significantly higher in grade $\mathrm{I}-\mathrm{V}(\mathrm{p}<0.05)$.

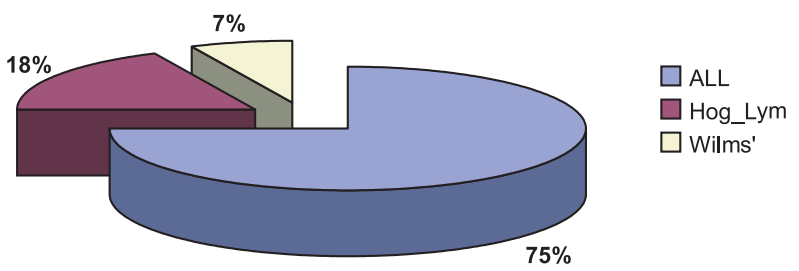

Fig: - 1. Distribution of types of neoplasm among the study population

Table-I

Frequency of psychiatric disorders according to broad diagnostic categories

\begin{tabular}{lc}
\hline Diagnostic category & Total \\
& $\mathrm{N}=32(\%)$ \\
\hline Any Disorder & $32(53.3)$ \\
Emotional Disorder & $28(87.5)$ \\
Behavioral Disorder & $11(34.7)$ \\
\hline
\end{tabular}

*Co-morbidity was considered.

Table-II

Frequency of specific psychiatric disorder

\begin{tabular}{lc}
\hline ICD-10 Diagnosis & $\begin{array}{c}\text { Total } \\
\mathrm{N}=60(\%)\end{array}$ \\
\hline Any Disorder & $32(53.3)$ \\
Any Emotional disorder & $28(87.5)$ \\
Separation anxiety disorder(SAD) & $02(7.1)$ \\
Specific phobia & $07(25.0)$ \\
Social phobia & $02(7.1)$ \\
Obsessive compulsive disorder(OCD) & $01(3.5)$ \\
Generalized anxiety disorder(GAD) & $04(14.3)$ \\
Major depressive disorder(MDD) & $04(14.3)$ \\
Dysthymic disorder & $01(3.5)$ \\
Other anxiety disorder & $07(25.0)$ \\
Any Behavioural disorder & $11(34.7)$ \\
Oppositional defiant disorder & $04(36.3)$ \\
Conduct disorder & $02(18.1)$ \\
Hyperkinetic disorder & $05(45.4)$ \\
\hline
\end{tabular}

*Co-morbidity was considered 


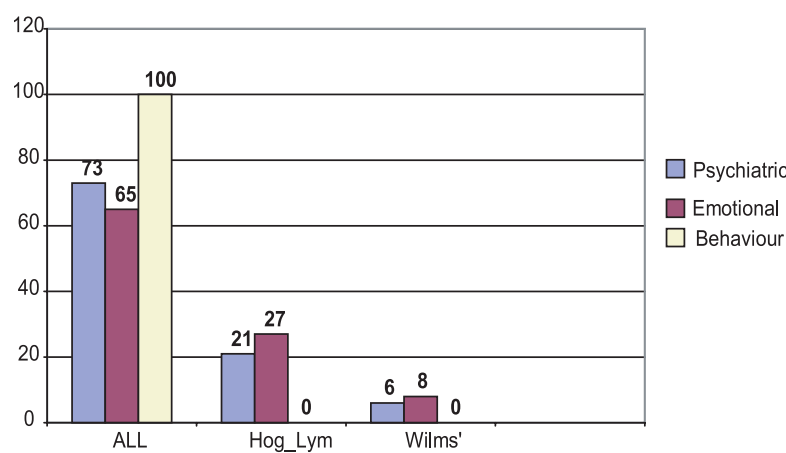

Fig.-2: Relationship between psychiatric disorder and types of neoplasm

Table-III

Relationship between Psychiatric diagnosis and different socio-demographic characteristics

\begin{tabular}{lccc}
\hline $\begin{array}{l}\text { Socio-demographic } \\
\text { variablesSex }\end{array}$ & \multicolumn{2}{c}{ Psychiatric diagnosis } & P value \\
Absent (\%) & Present (\%) & NS \\
\hline Boy & $15(53.6)$ & $19(59.4)$ & \\
Girl & $13(46.4)$ & $13(40.6)$ &
\end{tabular}

Age(in years)

5-10

19(67.9)

13(40.6)

$11-16$

09(32.1)

19(59.4)

Residence

Urban

$19(67.9$

19(59.4)

Rural

9(32.1)

13(40.6)

Family pattern

Nuclear

18(64.3)

17(53.1)

Joint

10(35.7)

15(46.9)

Economical status

Low

10(35.7)

11(34.4)

Middle

16(57.1)

15(46.9)

High

02(7.1)

06(18.8)

Level of education

0.012

Not yet started-

\begin{tabular}{lll} 
Preprimary & $07(25.0)$ & $02(06.3)$ \\
Grade I-V & $19(67.9)$ & $19(59.4)$ \\
Grade VI-XI & $02(07.1)$ & $11(34.4)$ \\
\hline
\end{tabular}

NS: not significant

\section{Table-IV}

Relationship between the broad category of Psychiatric disorder and socio-demographic characteristics

\begin{tabular}{lcccc}
\hline $\begin{array}{l}\text { Socio-demographic } \\
\text { variables }\end{array}$ & \multicolumn{4}{c}{ Psychiatric diagnosis } \\
\cline { 2 - 5 } & $\begin{array}{c}\text { Emotional disorder } \\
\text { N=28 }\end{array}$ & $\begin{array}{c}\text { Behavioral disorder } \\
\text { N=-11 }\end{array}$ \\
\hline Gender & $\begin{array}{c}\text { Present } \\
(\%)\end{array}$ & $\begin{array}{c}\text { p } \\
\text { value }\end{array}$ & $\begin{array}{c}\text { Present } \\
(\%)\end{array}$ & $\begin{array}{c}\text { p } \\
\text { value }\end{array}$ \\
\hline Boy & $13(46.4)$ & NS & $09(81.8)$ & 0.005 \\
Girl & $15(53.7)$ & & $02(18.2)$ & \\
Age(in years) & & & & \\
5-10 & $16(57.1)$ & NS & $06(54.6)$ & NS \\
11-16 & $22(42.9)$ & & $05(45.4)$ & \\
Level of education & & & & \\
Not yet started- & & & & \\
Preprimary & $06(21.4)$ & & $01(09.1)$ & \\
Grade I-V & $12(42.9)$ & 0.05 & $08(72.7)$ & 0.05 \\
Grade VI-XI & $09(35.7)$ & & $02(18.2)$ & \\
\hline
\end{tabular}

NS: not significant

\section{Discussion}

In our study, the most prevalent neoplasm was found ALL (75\%) followed by $18 \%$ with Hodgkin lymphoma and $6.7 \%$ with Wilms' tumor. ALL is the most common type of leukemia in pediatric group .Four out of every 100,000 children in the United States have ALL; with peak occurrence from age three to six. Lymphomas account for $10 \%$ of all childhood cancers. Wilms' Tumor, or cancer of the kidney, accounts for $6 \%$ of childhood cancers. Its peak incidence is in children age two to three ${ }^{1}$. These findings are more or less consistent with the present findings of the childhood neoplasm.

NS In a community based epidemiological study ${ }^{7}$ in Bangladesh the prevalence of psychiatric disorder was found $15.2 \%$. In broad category emotional disorders was 8.1\% followed by behavioral disorders was $10.9 \%$.Behavioral and emotional disorders occur frequently in general population of children. Around 4-8\% child and adolescents have clinically significant anxiety disorders that cause substantial distress or interfere markedly with everyday life. This makes anxiety disorders the second commonest group of child psychiatric disorders (second only to behavioral disorders and ahead of hyperactivity and depressive disorders) ${ }^{9}$. In the present study, out of 60 cases $53.3 \%$ had been suffering from psychiatric 
disorders (emotional $87.5 \%$ and behavioral disorders $34.7 \%$ ) which is 3-4 times more than that of general population.

Some chronic physical illness and their treatment have psychological consequences for the child. In the famous Isle of Wight study among children, the prevalence of psychiatric disorder was only slightly increased with physical illnesses that did not affect the brain. However, the prevalence was considerably higher with the organic brain disorder or epilepsy ${ }^{10}$. From this point of view, the high percentage of psychiatric disorders found in present study population is consistent with other study findings 4 , 10 . In the present study $73 \%$ of children with ALL suffered from psychiatric disorder and emotional and behavioral disorder were more in ALL case. This finding is consistent with the findings of the similar studies ${ }^{1,4}$.

In this study, boys suffered more psychiatric problem than that of girls and behavioral disorder was found significantly higher among boys. A landmark study finding was that the rate of psychiatric disorders in boys being twice that in girls ${ }^{7}$. It is also reported that behavioral problems are male excess disorder which was also consistent with other study result. While child mental health services tend to see more boys than girls, epidemiological studies do not show marked gender differences in overall rate of psychiatric disorders. Overall, higher rate of psychiatric morbidity was significantly associated with upper grade of education found in the present study. Chronic illnesses may impair reading ability and general intellectual development. It may affect self-esteem and the ability to form relationships. These consequences may persist into adult life ${ }^{7}$. Children with leukemia report missing up to 1020 weeks of school in one year, and as a result, many children repeat grades ${ }^{1}$. Therefore, these findings may guide care givers, teachers, and physicians to become more careful about them.

In our knowledge, this is the first study in Bangladesh to explore psychiatric problem among children and adolescents with neoplasm using sound methodology. However, the study has several limitations. The sample size was small. The subjects were referred to psychiatric department and there was possibility of having more psychiatric problem among them. Therefore, sample biasness exists and generalization of findings of the study is not possible.

\section{Conclusion:}

The identification and management of the emotional and behavioral disorders that accompany chronic physical illness including neoplasm would optimize treatment outcome and quality of life. Multi-centered broad based study with inclusion of large sample size only can confirm the findings of the present study.

\section{References:}

1. KC Chaudhuri Foundation, Springer India. Psychological consequences of chronic physical illnesses in children and adolescents. Indian J Pediatr 2002; 69:145-48.

2. Bleyer WA. The impact of childhood cancer on the United States and the World. Cancer J Clin 1990; 40: 355-67.

3. Sharan P, Mehta M, Choudhry VP. Psychiatric morbidity in children suffering from acute lymphoblastic leukemia. Pediatr Hematol Oncol 1999; 16:49-54.

4. Sharan P, Mehta M, Choudhry VP. Psychiatric disorders among parents of children suffering from acute lymphoblastic leukemia. Pediatr Hematol Oncol 1999; 16: 43-7.

5. Iqbal A, Siddiqui KS. Depression among parents of children with acute lymphoblastic leukemia. J Ayub Med Coll Abottabad 2002; 14:6-9.

6. Goodman R, Ford T, Richards H, Meltzer H, Gatward R. The Development and Well-Being Assessment: description and initial validation of an integrated assessment of child and adolescent psychopathology. J Child Psychol Psychiatry 2000; 41:645-57.

7. Mullick MSI, Goodman R. The prevalence of psychiatric disorders among 5-10 years olds in rural, urban and slum areas in Bangladesh: an exploratory study. Soc Psychiatr Epidemiol 2005; 40:663-71.

8. World Health Organization. The ICD-10 Classification of mental and behavioral disorders diagnostic criteria for research. World Health Organization, 1993.

9. Goodman R, Scott S .Child Psychiatry. $2^{\text {nd }}$ ed.Oxford: Blackwell Publishing; 2005.84p.

10. Gelder M, Harrison P, Cowen P. Shorter Oxford Textbook of Psychiatry. $5^{\text {th }}$ ed. New York. Oxford University Press; 2006.650-693p. 